\title{
Forward recursion approach of electromagnetic wave propagation characteristics in a slab of inhomogeneous magnetoplasma
}

\author{
A. M. Al-Khateeb, M. A. Khasawneh, and M. S. Bawa'aneh* \\ Department of Physics, \\ Yarmouk University, Irbid, Jordan \\ *corresponding author, E-mail: msbawaaneh@yu.edu. jo
}

\begin{abstract}
Propagation characteristics of electromagnetic radiation incident on an inhomogeneous magnetoplasma slab near a good conducting metallic surface is investigated. The inhomogeneous plasma slab is divided into thin layers (subcells) in order to allow for treating each plasma sub-cell as a homogeneous medium. A global matrix is formed upon matching the fields at all interfaces, which allows for the analytical determination of the reflection, absorption and transmission coefficients. For matching the tangential fields at the metallic surface, an impedance (Leontovich) boundary condition has been used. Propagation characteristics are calculated numerically for a set of parameters that may be suitable for many applications including stealth plasma. Numerical results show resonant absorption peaks near the electron cyclotron frequency that increase by increasing the equilibrium plasma density. They also show absorption enhancement by increasing the plasma slab width.
\end{abstract}

\section{Introduction}

Electromagnetic wave propagation characteristics in a plasma slab has for decades attracted the attention of researchers due to its versatile applications in different fields that is made possible mainly due to the wide range of possible plasma parameters. Such versatile applications include, but not limited to, atmospheric and space studies [1]-[9], telecommunications [10]-[18], antennas [19]-[21], material switches, absorbers and wave guides [22]-[27], and stealth technology [28]-[37], where the plasma is preferred as a good absorber or scatterer of EM-radiation. For example a good absorption window within the radar frequency range is desired in stealth plasma applications, while the undesired absorption of the $L$-band frequency range by the ionosphere layer may lead to positioning iono-error of above five meters in the GPS applications.

Electromagnetic waves with angular frequency $\omega$ can only propagate in plasma where $\omega>\omega_{p}$, the plasma frequency that is proportional to the square root of the refractive index of the plasma. If $\omega$ does not exceed $\omega_{p}$, then the EM-wave becomes evanescent after being reflected at the bulk plasma frequency, a process that gives rise to plasma heating [38], [39]. Within the framework of the AppletonHartree theory, the plasma ion motion is ignored and ions are treated as a heavy, neutralizing positive background compared to electrons; and the plasma is simplified to a dielectric material with all the electron and ion motion is accounted for in the plasma dielectric function [40]-[42].

Over the past years, researchers, targeting different applications regarding absorption and scattering characteristics of EM-waves in plasma, have studied various plasma regimes that included a plasma layer with different characteristics. Valagiannopoulos and Uzunoglu [2] used a model based on a 2D, double-layered planar waveguide restricted by two metallic surfaces, the upper layer of which possesses a step discontinuity, and characterized signal propagation within terrestrial environment. A thin inhomogeneous strip located inside a lossy homogeneous earth which scatters the excitation wave in the presence of a perfectly conducting ionospheric step discontinuity. C. Thoma et. al., [4] investigated radio blackout reentry conditions and obtained theoretical transmission coefficients of longitudinal EM-waves in a magnetized collisional plasma layer. They found out that a magnetic field of the order of a few hundred Gauss is required for the propagation of a $1 \mathrm{THz}$ frequency plane wave in the earth's upper ionosphere. In another study [5], Valagiannopoulos and Uzunoglu demonstrated the possibility of developing an imaging method for the underground environment based on the measurement of scattered extremely low frequency fields (ELF) by underground formations. The model assumes that the Earth-ionosphere structure can be approximated by a parallel-plate waveguide with perfectly conducting sidewalls. Lee et. al., [8] used predicted values of space weather indices from an established relationship between ionospheric threats and the space weather indices, that can be used to adjust the ionospheric threat model in real time. Zhang et. al., [9] studied the interaction of terahertz waves with an inhomogeneous plasma slab using the SMM method, where different density profiles were used. They found maximum absorbance occurring for homogeneous density profiles. Tian et. al., [12] investigated the effect of collision frequency and magnetic field amplitude on THz EM-wave propagation in a magnetized, collisional plasma slab. Chen Cong et. al., [15] investigated EM-wave characteristics in a plasma sheath forming during spacecraft reentry, where heperbolic plasma density profile is used. Bawaaneh et. al., [34, 35] have modified the long existing Appleton-Hartree magne- 
toionic dispersion relation of EM-wave propagation in cold, magnetized plasma to account for the thermal effect. They also calculated reflection, absorption and transmission coefficients of transverse EM-waves in a magnetized, collisional, inhomogeneous plasma layer near a metallic wall using the SMM method. They also used a kinetic model to study the characteristics of EM waves in hot plasma [36].

Matrix formalism of EM-wave characteristics in inhomogeneous plasma is a rigorous method to overcome the inhomogeneity in the plasma slab [see for example Refs. $[28,30,34]$ on the SMM method]. Properties of great influence on the propagation characteristics, such as the medium inhomogeneity, can be modeled by replacing an inhomogeneous layer by a succession of very thin discrete homogenous sub-layers. In the present work, the Forward Recursion Method is a matrix method derived from first principles, namely, the continuity of the tangential electromagnetic field components obtained from the Maxwell's curl equations. Using the boundary conditions for the EMfields, forward and backward fields on one side of a specific interface are related to those on the other side. The input to output relationship is, as usual, expressed in terms of a $2 \times 2$ matching matrix. In the presence of several interfaces, forward and backward fields will be propagated from one interface to the next with the help of such a $2 \times 2$ propagation matrix. The combination of a matching and a propagation matrix relating the fields across different interfaces is usually referred to as a transfer or transition matrix.

The paper is organized as follows; In Section 2, the forward recursion model equations are presented and the reflection, absorption and transmission coefficients are derived. Numerical results and their discussion are presented in Section 3, and conclusions are presented in Section 4.

\section{Forward recursion model equations}

Assume a linearly polarized plane wave such that $\vec{E}(x, t)=$ $\hat{z} E_{z}(x, t)$ is incident on an inhomogeneous magnetoplasma slab extending from $x=0$ to $x=a$. The incident, reflected and transmitted waves are propagating along $x$ parallel to a background uniform magnetostatic field $\vec{B}=\hat{x} B_{0}$, as shown in Figure 1. The conducting material at $x=b$ is aluminum. For $x<0$ the surrounding region of incidence has a permittivity $\epsilon^{(L)}=\epsilon_{0} \varepsilon^{(L)}(\omega)$, while it is $\epsilon^{(R)}=\epsilon_{0} \varepsilon^{(R)}(\omega)$ in the medium to the right of the plasma slab interface at $x=a$. The region to the right of the plasma slab for $a<x<b$ has a width $d=b-a$ and is expected to support the formation of standing wave patterns due to the interference of waves reflected from the good conductor at $x=b$ and those transmitted from the plasma interface at $x=a$. We divide the plasma slab into $n$ homogeneous layers with $m^{\text {th }}$ layer width of $d_{m+1}-d_{m}$, where $m=1,2,3, \ldots, n$. The time-harmonic electric field in each region of interest, as shown in figure 1 , are $E_{z}^{(L)}$ in the Lregion of incidence, $E_{z}^{(m)}$ in the $m^{\text {th }}$ plasma sub-cell, and $E_{z}^{(R)}$ in the R-region between $x=a$ and $x=b$. These

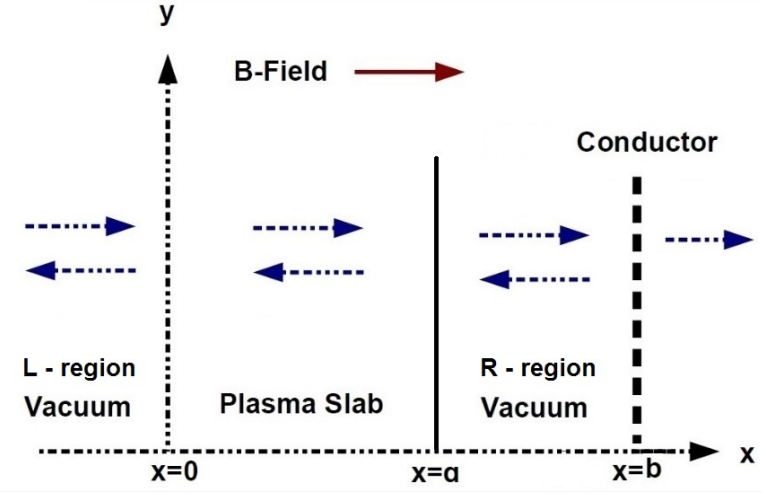

Figure 1: Geometry of the problem.

fields are given by

$$
\begin{aligned}
& E_{z}^{(L)}(x, t)=E_{0}\left(e^{-j k_{x}^{(L)} x}+A e^{j k_{x}^{(L)} x}\right) e^{j \omega t} \\
& E_{z}^{(m)}(x, t)=E_{0}\left(B_{m} e^{-j k_{x}^{(m)} x}+C_{m} e^{j k_{x}^{(m)} x}\right) e^{j \varphi}(2) \\
& E_{z}^{(R)}(x, t)=E_{0}\left(D e^{-j k_{x}^{(R)} x}+F e^{j k_{x}^{(R)} x}\right) e^{j \omega t}
\end{aligned}
$$

where $E_{0}$ is the amplitude of the incident field, and $A$ is the global reflection coefficient of the region of incidence, and $k_{x}^{(L, R)}=(\omega / c) \sqrt{\varepsilon^{(L, R)}(\omega)}$ the wave propagation constant in the region of incidence $(\mathrm{L})$ and in the region to the right of the plasma slab (R). The variation of the wave propagation constant over the $m^{\text {th }}$ homogeneous plasma sub-cell has been ignored with $B_{m}$ and $C_{m}$ being the partial transmission and reflection coefficients, respectively. Similarly, the constants $D$ and $F$ stand for the partial transmission and reflection coefficients in the R-region, respectively.

At the conducting wall at $x=b$ we introduce an impedance (Leontovich) boundary condition such that $E_{z}(b, t)=-Z_{c} H_{y}(b, t)$, where $Z_{c}$ is the characteristic impedance of the conducting surface, and the tangential magnetic field is $H_{y}=-\left(j / \mu_{0} \omega\right) \partial E_{z} / \partial x$. Accordingly, the relation between $D$ and $F$ is established and the electric field $E_{z}^{(R)}$ of equation (3) takes on the following form;

$$
\begin{aligned}
& E_{z}^{(R)}=-j 2 E_{0} D U \sin k_{x}^{(R)}(x-b) e^{j \omega t-j k_{x}^{(R)} b} \\
& U=\frac{\eta^{(R)}-Z_{c}}{\eta^{(R)}+Z_{c}}, \quad \eta^{(R)}=\frac{\mu_{0} \omega}{k_{x}^{(R)}}=\frac{Z_{0}}{\sqrt{\varepsilon^{(R)}(\omega)}}, \\
& Z_{c}=\sqrt{\frac{\mu}{\epsilon}}=\sqrt{\frac{\mu_{0}}{\epsilon_{0}-j \frac{S}{\omega}}}=\frac{Z_{0}}{\sqrt{\varepsilon^{(c)}(\omega)}}, \\
& \varepsilon^{(c)}(\omega)=1-j \frac{S}{\epsilon_{0} \omega}, \quad Z_{0}=\sqrt{\frac{\mu_{0}}{\epsilon_{0}}}=377 \Omega(7)
\end{aligned}
$$

where $\eta^{(R)}$ is the impedance of the R-region, $Z_{0}$ is the vacuum impedance, $\varepsilon^{(c)}(\omega)$ is the dielectric function of the metallic surface at $x=b, \varepsilon^{(R)}(\omega)$ the dielectric function of the R-region which is equal to one for vacuum and $S$ is the electric conductivity of the metallic wall,

The integration constants $A, B_{m}, C_{m}$ and $D$ are determined by matching the tangential electric field $E_{z}$ at $x=0$, 
$x=a$, and at all interfaces between adjacent layers within the plasma slab. Consequently, we obtain the following expressions for the integration constants $A$ and $D$;

$$
\left[\begin{array}{l}
1 \\
A
\end{array}\right]=\left[\begin{array}{ll}
T_{11} & T_{12} \\
T_{21} & T_{22}
\end{array}\right]\left[\begin{array}{l}
Q_{1} \\
Q_{2}
\end{array}\right] D,
$$

where $Q_{1}$ and $Q_{2}$ are given by

$$
\begin{aligned}
Q_{1} & =q_{0}\left[j k_{x}^{(n)} \sin \left(k_{x}^{(R)} d\right)+k_{x}^{(R)} \cos \left(k_{x}^{(R)} d\right)\right](9) \\
Q_{2} & \left.=q_{0}\left[j k_{x}^{(n)} \sin \left(k_{x}^{(R)} d\right)-k_{x}^{(R)} \cos \left(k_{x}^{(R)} d\right)\right] 0\right) \\
q_{0} & =\frac{\eta^{(R)}-Z_{c}}{\eta^{(R)}+Z_{c}} \frac{e^{j k_{x}^{(n)} a-j k_{x}^{(R)} b}}{k_{x}^{(n)}}
\end{aligned}
$$

and the coefficients $T_{11}, T_{12}, T_{21}, T_{22}$ are determined as follows;

$$
\begin{aligned}
& T=T_{1}\left(\prod_{m=2}^{m=N} T_{m}\right) \equiv\left[\begin{array}{ll}
T_{11} & T_{12} \\
T_{21} & T_{22}
\end{array}\right] \\
& T_{1}=\frac{1}{2 k_{x}^{(L)}}\left[\begin{array}{cc}
k_{x}^{(L)}+k_{x}^{(1)} & k_{x}^{(L)}-k_{x}^{(1)} \\
k_{x}^{(L)}-k_{x}^{(1)} & k_{x}^{(L)}+k_{x}^{(1)}
\end{array}\right]
\end{aligned}
$$

where the matrix $T_{m}$ of the $m^{\text {th }}$ plasma sub-cell for $2 \leq$ $m \leq N$ is given by

$$
\begin{aligned}
& T_{m}=\frac{1}{2 k_{x}^{(m-1)}}\left[\begin{array}{ll}
\alpha_{11} & \alpha_{12} \\
\alpha_{21} & \alpha_{22}
\end{array}\right], \\
& \alpha_{11}=\left[k^{(m-1)}+k^{(m)}\right] e^{j d_{m}\left(k^{(m-1)}-k^{(m)}\right)} \\
& \alpha_{12}=\left[k^{(m-1)}-k^{(m)}\right] e^{j d_{m}\left(k^{(m-1)}+k^{(m)}\right)} \\
& \alpha_{21}=\left[k^{(m-1)}-k^{(m)}\right] e^{-j d_{m}\left(k^{(m-1)}+k^{(m)}\right)} \\
& \alpha_{22}=\left[k^{(m-1)}+k^{(m)}\right] e^{-j d_{m}\left(k^{(m-1)}-k^{(m)}\right)}
\end{aligned}
$$

Also $k_{x}^{(m)}=(\omega / c) \sqrt{\varepsilon^{(m)}(\omega)}$, where $\varepsilon^{(m)}$ is the dielectric constant of the $m^{t h}$ sublayer of plasma given for parallel propagation in collisional, cold, magnetized plasma by [41]-[43]

$$
\varepsilon_{ \pm}^{(m)}=1-\frac{\omega_{p e, m}^{2}}{\omega\left(\omega \mp \omega_{c e}-j \nu\right)}
$$

Here $\omega_{p e, m}$ is the $m^{\text {th }}$ layer electron plasma frequency and $\nu$ is the collision frequency. The upper $(+)$ and lower $(-)$ signs in $\varepsilon_{ \pm}^{(m)}$ correspond, respectively, to left $(L)$ and right $(R)$ hand polarized transverse waves in plasma. In the literature, one may find various formulae for the dielectric function characterizing various plasma regimes; see for example [43]-[49].
The global transmission and reflection coefficients can be obtained from equation (8), namely,

$$
\begin{aligned}
\tau & =\frac{\eta^{(R)}}{Z_{c}} D=\frac{\eta^{(R)}}{Z_{c}} \frac{1}{T_{11} Q_{1}+T_{12} Q_{2}}, \\
\rho & =A=\frac{T_{21} Q_{1}+T_{22} Q_{2}}{T_{11} Q_{1}+T_{12} Q_{2}}
\end{aligned}
$$

Hence, reflectance $(R)$, transmittance $(T)$ and absorbance $(A)$ are then given by

$$
\begin{aligned}
& \mathrm{T}=\tau \tau^{*}, \quad \mathrm{R}=\rho \rho^{*}, \\
& \mathrm{~A}=1-\mathrm{R}-\mathrm{T}
\end{aligned}
$$

\section{Numerical results and discussion}

In this section, we present numerical examples on the impact of varying the inhomogeneous magnetoplasma slab parameters on reflection, absorption and transmission of a transverse electromagnetic waves incident upon the plasma. We consider an inhomogeneous plasma slab with exponentially increasing density profile such that $n=n_{0} e^{\alpha x}$, where $n_{0}$ is the maximum density of the plasma at $x=a$, $\alpha$ being a positive constant chosen such that the plasma density ranges exponentially from one tenth of $n_{0}$ to $n_{0}$ within the thickness of the plasma slab that is stratified into $N$ homogeneous sub-layers (see figure 1). We treat

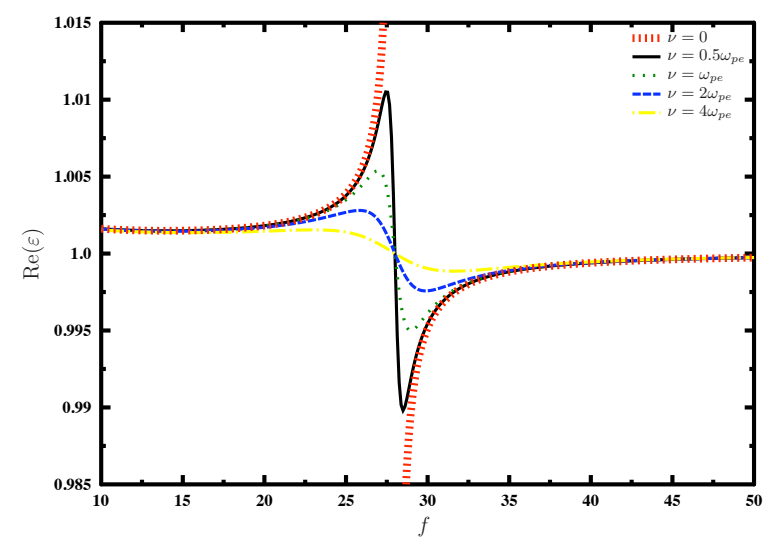

Figure 2: Real part of the dielectric function versus wave frequency $(\mathrm{GHz})$ for different collision frequency values. $n_{0}=10^{17} \mathrm{~m}^{-3}, f_{c e}=\omega_{c e} / 2 \pi=28 \mathrm{GHz}$.

the plasma as a Lorentz gas of non-interacting electrons and immobile neutralizing background of positive ions immersed in an externally applied uniform magnetostatic field $\vec{B}_{0}=B_{0} \hat{x}$. For wave propagation along the applied magnetic field, a magnetoplasma of cold electrons and cold ions supports three wave modes, namely, a longitudinal electron plasma oscillation (not affected by the magnetic field) and two transverse right- and left-circularly polarized electromagnetic waves. Left circularly polarized waves [positive 
sign of equation 15] are not resonant with the electron motion. We consider the right polarized waves only.

For collisionless plasma, where the plasma dielectric function is represented by equation 15 with ignoring the collisional frequency, EM-waves have two propagation intervals in the frequency range interrupted by a stop band of frequency $\omega \in\left(\omega_{c e}, \omega_{R}\right)$, where $\omega_{R}=\omega_{c e} / 2+$ $1 / 2 \sqrt{\omega_{c e}+4 \omega_{p e}}[36,43]$. The presence of collision, however, narrows the stop band till it is bridged completely above a certain collision frequency making the real part of the dispersion function of equation 15 , hence the phase velocity, a continuous function of the wave frequency; higher collision frequency values reduce the peaks toward $\omega \approx c k$ value. The real part of the dielectric function versus the wave frequency is shown in figure 2 for different collisional frequency values. Here, $f_{c e}=\omega_{c e} / 2 \pi=28 G H z$ corresponding to $B_{0}=1$ Tesla and $n_{0}=10^{17} \mathrm{~m}^{-3}$.

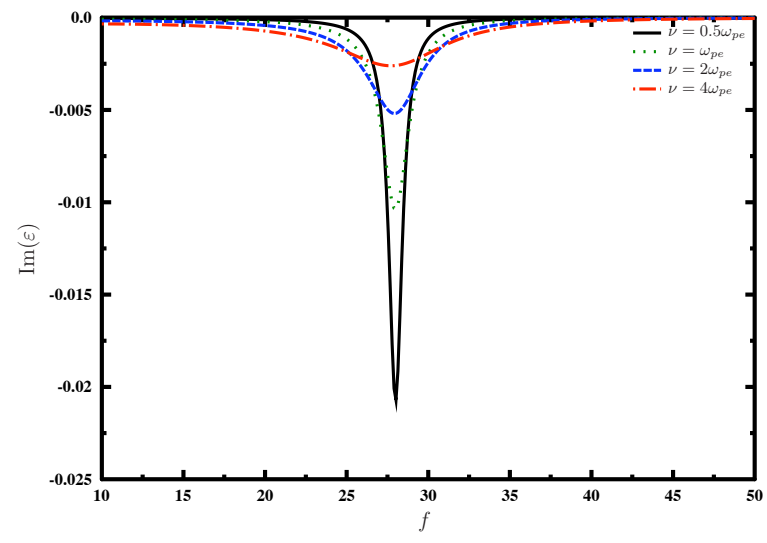

Figure 3: Imaginary part of the dielectric function versus wave frequency $(\mathrm{GHz})$ for different collision frequency values. $n_{0}=10^{17} \mathrm{~m}^{-3}, f_{c e}=\omega_{c e} / 2 \pi=28 \mathrm{GHz}$.

Collision of equation 15 gives the dielectric function an imaginary part providing a mechanism for wave absorption within the plasma slab. Figure 3 shows the imaginary part of the dielectric function for different values of collision frequency and the same values of equilibrium density and cyclotron frequency as in figure 2. As expected, the function peaks at the cyclotron frequency, hence we should expect high absorption rates in the vicinity of $\omega_{c e}$.

In figures 4 to 7 , we present numerical results of characterization of EM-wave propagation in the plasma slab described in Fig. 1 for a set of parameters that may be useful in stealth plasma application. Fig. 4 shows the absorbance versus wave frequency $(\omega)$ for different plasma interface densities $n_{0}$. Parameters used to produce the figure are $a=5$ $\mathrm{cm}, b=7 \mathrm{~cm}$ (width of the R-region is $d=b-a=2 \mathrm{~cm}$ ), cyclotron frequency $f_{c e}=\omega_{c e} / 2 \pi=28 \mathrm{GHz}$ corresponding to $B_{0}=1$ Tesla, and aluminum is used as the metallic conductor at $x=b$. The figure shows resonant absorption peaks at wave frequency in the vicinity of the electron

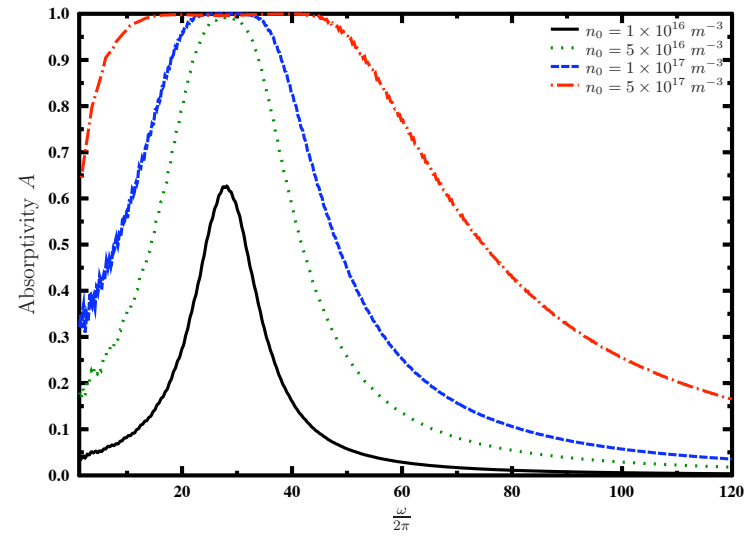

Figure 4: Absorbance versus wave frequency $(\mathrm{GHz})$ for different plasma interface densities $n_{0}, f_{c e}=\omega_{c e} / 2 \pi=28$ $\mathrm{GHz}$.

cyclotron frequency. This peak saturates to full absorption and broadens on the wave frequency range as the plasma density increases. This is well understandable since the higher the plasma density the less transparent it becomes for a specific wave frequency.

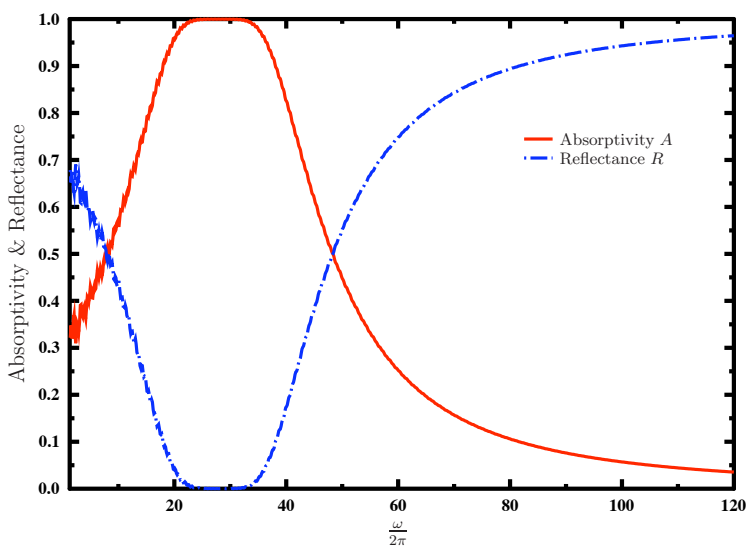

Figure 5: Absorbance and reflectance versus wave frequency $(\mathrm{GHz}), f_{c e}=\omega_{c e} / 2 \pi=28 \mathrm{GHz}$.

The reflectance versus wave frequency curves are the up side down inverted image of the absorptivity curve since we have a negligible wave transmission beyond $x=b$ due to reflection from the good conducting metallic surface. Result is shown in Fig 5 for reflected and absorbed signals versus the wave frequency for $n_{0}=10^{17} \mathrm{~m}^{-3}$ and the same parameters of Fig. 4, where the peak corresponds to absorption and the trough corresponds to reflection. As expected, absorbance is the flipped curve of reflectance since transmittance is negligible due to the presence of the metal to 


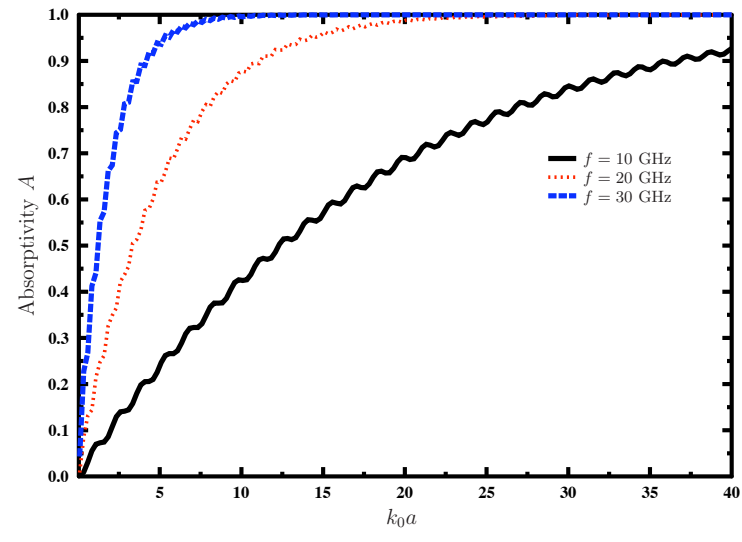

Figure 6: Absorbance versus $k_{0} a$ for three different wave frequencies, $f_{c e}=28 \mathrm{GHz}$.

the right.

Fig. 6 shows the absorbance versus the normalized plasma slab width $k_{0} a=\frac{2 \pi a}{\lambda_{0}}$ for different wave frequencies $(f=\omega / 2 \pi)$, where $\lambda_{0}$ is the wavelength of the incident wave. The figure shows increase in absorptivity as the plasma slab width increases to reach the full absorption saturation plateau after a certain slab width. The figure also shows faster absorption saturation as the wave frequency gets closer to the electron cyclotron frequency $f_{c e}=28 \mathrm{GHz}$, as expected; for the almost resonant wave frequency of $f=30 \mathrm{GHz}$, the curve reaches full absorption near $k_{0} a \approx 10$ corresponding to a plasma slab width of as small as $1.6 \lambda_{0}$. Fig. 7 shows the absorbance versus the cyclotron frequency $f_{c e}$ for three different wave frequencies. For each curve, we see full absorption in the vicinity of the cyclotron resonant frequency $f_{c e}=f_{\text {wave }}$, namely at $f_{\text {wave }}=30,40,50 \mathrm{GHz}$. The width of the peak is determined by the plasma density [see Fig. 4].

\section{Conclusion}

In this paper we present analytical and numerical investigation of the problem of reflection, absorption and transmission of a transverse electromagnetic wave incident upon a nonuniform, magnetized plasma slab near a good conducting metallic wall with wave propagation along a constant magnetic field as shown in figure 1. The presence of the conducting surface at $x=b$ allows for the formation of standing waves and the modification of the total reflection and transmission coefficients. Derived from basic principles of the continuity of the tangential field components in the Maxwell's curl equation, a forward recursion method is used to obtain a matrix of global reflection and transmission coefficients by applying the suitable boundary conditions. The resulting transition matrix is then used to obtain reflectance, transmittance and absorptivity analytically and numerically. Numerical investigation shows absorption rate

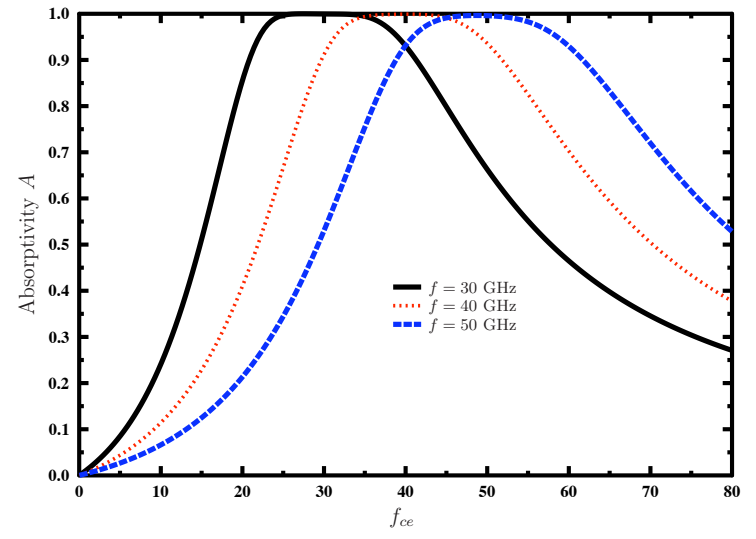

Figure 7: Absorbance versus cyclotron frequency $f_{c e}=$ $\omega_{c e} / 2 \pi$ for three different wave frequencies, $n_{0}=$ $10^{17} \mathrm{~m}^{-3}$.

peaks in the vicinity of the electron cyclotron frequency that are enhanced by the increase in equilibrium plasma density, where the plasma transparency to EM-waves decreases, hence absorption increases. The numerical results also show absorption enhancement as the plasma slab width is increased. Compared with numerical results obtained using the scattering matrix method (See for example Ref. [34]), results are in qualitative and quantitative agreement. The present technique is easy to implement numerically.

\section{Acknowledgement}

MSB acknowledges the support from Yarmouk University (Fund No. 2017/23).

\section{References}

[1] V. N. Laxmi, A. K. Saha, Interaction of electromagnetic waves in the ionosphere, Radio Science, Vol. 23 (06), pp. 95167, 1988.

[2] C.A. Valagiannopoulos and N.K. Uzunoglu, Scattering of ELF waves by underground formations because of night-day ionospheric ridge, RADIO SCIENCE, VOL. 42, RS6S32, 2007.

[3] M. Keidar, M. Kim and I. Boyd, Analysis of an electromagnetic mitigation scheme for reentry telemetry through plasma, J. Spacecraft Rockets, 45(6), pp. 1223-1229, (2008).

[4] C. Thoma, D. V. Rose, C. L. Miller, R. E. Clark and T. P. Hughes, Electromagnetic wave propagation through an overdense magnetized collisional plasma layer, J. Applied Physics, Vol. 106, pp. 043301, (2009). 
[5] C.A. Valagiannopoulos and N.K. Uzunoglu, Simplified model for EM inverse scattering by longitudinal subterranean inhomogeneities exploiting the dawn/dusk ionospheric ridge, IIET Microw. Antennas Propag., Vol. 5, Iss. 11, pp. 13191327, 2011.

[6] Kai Li, Xue Yan Sun, Hou Tao Zhai, Propagation of ELF Electromagnetic Waves in the Lower Ionosphere, IEEE Transactions on Antennas and Propagation, Vol. 59 (2), pp. $661666,2011$.

[7] Rohtash Singh, A. K. Sharma and V. K. Tripathi, Grating formation by high power radiation in near equator ionosphere, Phys. Plasmas, Vol. 18, p. 112101, 2011.

[8] Jinsil Lee, Sam Pullen, Seebany Datta-Barua, and Jiyun Lee, Real-Time Ionospheric Threat Adaptation Using a Space Weather Prediction for GNSSBased Aircraft Landing Systems, IEEE Transactions on Intelligent Transportation Systems, Vol. 18(7), pp. 1752761, 2017.

[9] Youyi Zhang, Guanjun Xu and Zhengqi Zheng, Propagation of terahertz waves in a magnetized, collisional and inhomogeneous plasma with the scattering matrix method, Optik, Vol. 182, pp. 618 624, Apr. 2019.

[10] M. P. Bachynski, Electromagnetic Wave Penetration of Reentry Plasma Sheaths, RADIO SCIENCE Journal of Research NBS/USNC- URSI, Vol. 69D (2) (1965).

[11] J. P. Pybak and R. I. Churchil, Progress in reentry communications, IEEE Trans. Aerosp. Electron. Syst., vol. AES-7, no. 5, pp. 87994, 1971.

[12] Yunxian Tian, Weizhong Yan, Xiaoliang Gu, Xiaolin Jin, Jianqing $\mathrm{Li}$ and Bin Li, Effect of magnetized plasma on the propagation properties of obliquely incident $\mathrm{THz}$ waves, AIP Advances, Vol. 7, p. 125325$11,2017$.

[13] Jiyun Lee, Y. T. Jade Morton, Jinsil Lee, Hee-Seung Moon and Jiwon Seo, Monitoring and Mitigation of Ionospheric Anomalies for GNSS-Based Safety Critical Systems: A review of up-to-date signal processing techniques, IEEE Signal Processing Magazine, Vol. 34 (5), pp. 96 110, 2017.

[14] Xin Yang, Bing Wei and Weike Yin, A new method to analyze the EM wave propagation characteristics in the hypersonic sheath, Optik, Vol. 148, Pages 187195,2017

[15] Chen Cong, Cheng Shi and Zhang Shi, A comparative study on electromagnetic wave propagation in a plasma sheath based on double parabolic model, Optik, Vol. 159, Pages 69-78, 2018.
[16] Guanjun $\mathrm{Xu}$ and Zhaohui Song, Interaction of terahertz wave propagation in a homogeneous, magnetized and collisional plasma slab, Waves in Random and Complex Media, DOI: 10.1080/17455030.2018.1462542, Published online: 26 Apr 2018.

[17] Cagatay Tanil; Samer Khanafseh; Mathieu Joerger; Boris Pervan, An INS Monitor to Detect GNSS Spoofers Capable of Tracking Vehicle Position, IEEE Transactions on Aerospace and Electronic Systems, Vol. 54 (1), pp. 131-143, 2018.

[18] Kai Yuan, Jiamin Chen, Linfang Shen, Xiaohua Deng, Ming Yao, and Lujun Hong Impact of Reentry Speed on the Transmission of Obliquely Incident $\mathrm{THz}$ Waves in Realistic Plasma Sheaths, IEEE Transactions on Plasma Science, Vol. 46(2), pp. 373-379, 2018.

[19] Mohammad A Matin, Review on Millimeter Wave Antennas- Potential Candidate for 5G, ADVANCED ELECTROMAGNETICS, VOL. 5, NO. 3, Dec. 2016.

[20] S. Zainud-Deen, S. Abd Elhamied and H. Abd ElAzem Malhat, Integrated Millimeter-Wave Antennas for On-Chip Communication, ADVANCED ELECTROMAGNETICS, VOL. 5, NO. 1, Mar. 2016.

[21] G. G. Machado, M. T. de Melo, H. V. H. Silva Filho, A. G. Neto and T. R. de Souza, Low Radar Crosssection and Low Cost Dipole Antenna Reflector, ADVANCED ELECTROMAGNETICS, VOL. 6, NO. 3, Oct. 2017.

[22] Arvinder Singh and Navpreet Singh, Guiding of a laser beam in a collisionless magnetoplasma channel, Journal of the Optical Society of America B, Vol. 28, Number 8, pp. 1844-1850, 2011.

[23] MK Hedayati, et. al, Photodriven Super Absorber as an Active Metamaterial with a Tunable MolecularPlasmonic Coupling, Advanced Optical Materials, Vol. 2, Number 8, pp. 705-710, 2014.

[24] Mady Elbahri, et. al, Photoswitchable molecular dipole antennas with tailored coherent coupling in glassy composite, Light Science and Applications, Vol.4, e316, Jul. 2015.

[25] M. S. Bawa'aneh, Ahmed M. Al-Khateeb and YcGhim, Resistance and reactance of monopole fields induced by a test charge drifting off-axis in a cold and collisional cylindrical plasma, Chinese Physics Letters, Vol. 35, Number 8, pp. 085101-5, 2018.

[26] Shahin Homaeigohar and Mady Elbahri, Switchable Plasmonic Nanocomposites, Advanced Optical Materials, 7, 1801101 (133), 2019.

[27] Hodjat Hajian, Amir Ghobadi, Bayram Butun and Ekmel Ozbay, Active metamaterial nearly perfect light 
absorbers: a review, Journal of the Optical Society of America B, Vol. 36, Issue 8, pp. F131-F143, (2019).

[28] Mounir Laroussi and J. Reece Roth, Numerical calculation of the reflection, absorption and transmission of microwaves by a nonuniform plasma slab, IEEE Transactions on Plasma Science, Vol. 21, pp.366-372 (1993).

[29] M. Laroussi, Interaction of microwaves with atmospheric pressure plasmas, Int. J. Infrared Millim. Waves, Vol. 16, pp. 2069-2083, 1995.

[30] Bin Jie Hu, Gang Wei and Sheng Li Lai, SMM Analysis of Reflection, Absorption and Transmission from Nonuniform, Magnetized Plasma Slab, IEEE Transactions on Plasma Science, Vol. 27, pp.1131-1136 (1999).

[31] Andrei B. Petrin, On the transmission of microwaves through plasma layer, IEEE Transactions on Plasma Science, vol. 28, pp.1000-1008 (2000).

[32] D. L. Tang, A. P. Sun, X. M. Qiu and Paul K. Chu, Interaction of electromagnetic waves with a magnetized, nonuniform plasma slab, IEEE Transactions on Plasma Science, Vol. 31, pp.405-410 (2003).

[33] R. Singh and A. K. Sharma, anomalous absorption of a whistler in rippled density plasma, Phys. Scripta, Vol. 82 (1), p. 015503 (2010).

[34] M. S. Bawa'aneh, A. M. Al-Khateeb and A. S. Sawalha, Microwave propagation in a magnetized inhomogeneous plasma slab using the AppletonHartree magneto-ionic theory, Canadian Joural of Physics, Vol. 90, pp.241-247 (2012).

[35] M. S. Bawa'aneh, A. M. Al-Khateeb and A. S. Sawalha, Microwave Propagation in Warm, Collisional Magnetoionic Media, IEEE Transactions on Plasma Science, vol. 41, pp.2496-2500 (2013).

[36] M. S. Bawaaneh, A. M. Al-Khateeb, and Y.-C. Ghim, Thermal Enhancement of Absorption of EM Radiation in a Hot Magnetoplasma Slab, IEEE Transactions on Antennas and Propagation, Vol. 66, No. 12, pp. 6525 6530, 2018.

[37] Mahdi Rahmanzadeh et. al, Analytical Investigation of Ultrabroadband Plasma Graphene Radar Absorbing Structure, IEEE Transactions on Plasma Science, Vol. 45, pp. 945-954, (2017).

[38] A. D. Boardman, Electromagnetic Surface Modes (Wiley, New York, 1982).

[39] Yu. M. Aliev, H. Schlüter, and A. Shivarova, GuidedWave-Produced Plasmas (Springer, Berlin, 2000), p. 31 .
[40] I. P. Shkarofsky, Generalized Appleton-Hartree Equation for Any Degree of Ionization and Application to the Ionosphere. Proceedings of the IRE, Vol. 49 (12), pp. 1857-1871, 1961.

[41] Curtis C. Johnson, Field and Wave Electrodynamics (McGraw-Hill, New York, 1965).

[42] N.A. Kral and A.W. Trivelpiece, Principles of Plasma Physics (McGraw-Hill, 1973).

[43] D. B. Melrose, Instabilities is Space and Laboratory Plasma, Cambridge University Press, 1986.

[44] S. Peter Gary, Theory of Space Plasma Microinstabilities, Cambridge Atmospheric and Space Science Series, 2005.

[45] M. S. Bawa'aneh, Stimulated Brillouin scattering of $X$-waves in magnetized plasma, Journal of Plasma Physics, vol. 72, part 5, pp. 68797, 2006.

[46] M. S. Bawa'aneh and T. J. M. Boyd, Enhanced levels of stimulated Brillouin reflectivity from nonMaxwellian plasmas, Journal of Plasma Physics Vol. 73, No. 2, pp. 159-166, 2007.

[47] M. S. Bawa'aneh, G. Assayed and S. Al-Awfi, Filamentation Instability of Electromanetic Radiation in Magnetized Plasma, IEEE Transactions on Plasma Science, Vol. 38, No. 5, pp. 1066-1072, 2010.

[48] Alireza Paknezhad, Nonlinear Raman forward scattering of a short laser pulse in a collisional transversely magnetized plasma, Phys. Plasmas, Vol. 20, pp. 012110-1 - 012110-9, 2013.

[49] M. S. Bawa' aneh, Ghada Assayed, M. R. Said and S. Al-Awfi Convective laser filamentation instability in magnetized plasma, Canadian Journal of Physics, Vol. 92, pp. 504-508, 2014. 